\title{
Publisher Correction: Flooding risk: Wastewater disruptions
}

Monica Contestabile

Correction to: Nature Sustainability https://doi.org/10.1038/s41893-018-0056-6, published online 18 April 2018.

In the version of this Research Highlight originally published, the researcher's name was mistakenly spelled 'Hummer'; it should have been 'Hummel. This has now been corrected in all versions. 